\title{
New Nonlinear Controller for a Class of Chaotic Systems Based on Adaptive Backstepping Fuzzy-Immune Control
}

\author{
Kai-Shiuan Shih, ${ }^{1}$ Tzuu-Hseng S. Li, ${ }^{1}$ and Shun-Hung Tsai ${ }^{2}$ \\ 1 aiRobots Laboratory, Department of Electrical Engineering, \\ National Cheng Kung University, Tainan 70101, Taiwan \\ ${ }^{2}$ Graduate Institute of Automation Technology, National Taipei University of Technology, \\ Taipei 10608, Taiwan
}

Correspondence should be addressed to Shun-Hung Tsai, shtsai@ntut.edu.tw

Received 31 July 2010; Revised 15 March 2011; Accepted 28 March 2011

Academic Editor: E. E. N. Macau

Copyright (c) 2011 Kai-Shiuan Shih et al. This is an open access article distributed under the Creative Commons Attribution License, which permits unrestricted use, distribution, and reproduction in any medium, provided the original work is properly cited.

An adaptive backstepping fuzzy-immune controller for a class of chaotic systems is proposed. An adaptive backstepping fuzzy method and adaptive laws are used to approximate nonlinear functions and the unknown upper bounds of uncertainty, respectively. The proposed adaptive backstepping fuzzy-immune controller guarantees the stability of a class of chaotic systems while maintaining good tracking performance. The fuzzy-immune algorithm is used for mathematical calculations. The intelligence algorithm consists of the adaptive backstepping fuzzy method and a novel fuzzy-immune scheme which generates optimal parameters for the control schemes. Finally, two simulation examples are given to illustrate the effectiveness of the proposed approach.

\section{Introduction}

Adaptive fuzzy logic controllers provide a systematic and efficient framework for incorporating linguistic fuzzy information from human experts. In [1], an adaptive fuzzy logic control theory was derived for a class of uncertain nonlinear single-input single-output (SISO) systems. Moreover, many scientists have since dedicated a lot of effort to solving the adaptive fuzzy control problem of uncertain nonlinear systems [2-10]. Furthermore, the stability of uncertain nonlinear systems has been addressed by the integration of fuzzy logic control and the adaptive laws. [11-20]. Subsequently, several methodologies have been instituted for controlling nonlinear systems [20-29]. The primary advantage of adaptive fuzzy control scheme is insensitive to internal uncertainty and external disturbances. Adaptive fuzzy 
control approaches only can perform desired performance for a simple class of nonlinear systems. If nonlinear systems without satisfying the matching conditions, the adaptive fuzzy control methodologies cannot be implemented.

In the past decade, many adaptive fuzzy control schemes have been developed by combining the backstepping technique [30-32]. The primary advantage of adaptive backstepping fuzzy control is that the matching conditions are not needed. Backstepping is based on the nonlinear stabilization technique of adding an integrator. Adaptive backstepping fuzzy control schemes can provide a systematic framework for tracking or regulation strategies [33-40].

In the past decade, the research area of controlling chaos has received increasing attention. Chaos is a complex nonlinear dynamical system, and it is commonly difficult to exactly predict the behavior of a chaotic system. Recently, many successful methods for controlling chaos have been developed [3, 8]. In the present study, we propose an adaptive backstepping fuzzy-immune controller for a class of chaotic systems. Based on the backstepping algorithm, the fuzzy methodology augmented by an immune algorithm is proposed as a new evolution algorithm, which maintains the advantages of simplicity and easy handling. The four main contributions are (1) an adaptive backstepping fuzzy-immune tracking controller for a class of chaotic systems is proposed, (2) the controller does not require a priori knowledge of the sign of the control coefficient, (3) a novel fuzzy-immune algorithm is used to find the optimal solution, and (4) a correct term can be used to eliminate disturbance.

The rest of this paper is organized as follows. In Section 2, system statement and description of fuzzy systems for chaotic system are presented. The adaptive backstepping fuzzy controller technique and a novel fuzzy-immune mechanism are discussed in Section 3. The results of simulations for chaotic systems are presented to confirm the validity of the proposed control scheme in Section 4. Finally, the conclusions are given in Section 5.

\section{System Statement and Description of Fuzzy Systems for Chaotic System}

In this paper, we consider a class of chaotic systems that can be shown in strict-feedback systems with nonlinear functions and disturbances

$$
\begin{aligned}
\dot{x}_{1} & =x_{2}+d_{1}(t), \\
\dot{x}_{2} & =x_{3}+d_{2}(t), \\
& \vdots \\
\dot{x}_{n} & =f_{n}\left(\bar{x}_{n}(t)\right)+g_{n}\left(\bar{x}_{n}(t)\right) u(t)+d_{n}(t), \\
y & =x_{1},
\end{aligned}
$$

where $\bar{x}_{n}(t)=\left[x_{1}(t), \ldots, x_{n}(t)\right]^{T} \in R^{n}, f_{n}\left(\bar{x}_{n}(t)\right)$ and $g_{n}\left(\bar{x}_{n}(t)\right)$ are smooth functions, $u(t)$ and $y$ are control input and output variables, respectively. $d_{1}(t), \ldots, d_{n}(t)$ denote external disturbance. However, the bound of external disturbance is difficult to obtain in the practical applications. The control objective is to design a stabilizing controller for the system described 
by (2.1) so that the tracking error converges to zero asymptotically despite the presence of unknown nonlinearities and disturbances.

\subsection{Description of Fuzzy Systems}

Fuzzy logic systems have been successfully employed to approximate the mathematical models of nonlinear systems. The fuzzy systems can be divided into four parts: fuzzifier, fuzzy rule base, fuzzy inference engine, and defuzzifier. The fuzzy mechanism is described by IF-THEN rules from an input linguistic vector $\bar{x}_{n}(t)$ to an output variable $f\left(\bar{x}_{n}(t)\right)$

$$
\begin{aligned}
& R^{i}: \quad \text { If } x_{1} \text { is } F_{i 1}, \quad x_{2} \text { is } F_{i 2}, \ldots, x_{n} \text { is } F_{i n}, \\
& \text { then } y \text { is } G_{i}, \quad i=1,2, \ldots, m,
\end{aligned}
$$

where $m$ is the number of rules. $F_{i 1}, F_{i 2}, \ldots, F_{i n}$ and $G_{i}$ are the fuzzy sets and $x_{1}, x_{2}, \ldots, x_{n}$ are states of system. Using a singleton function, center average defuzzification, and product inference, the fuzzy systems output is

$$
\bar{f}\left(\bar{x}_{n}\right)=\frac{\sum_{i=1}^{m} \bar{y}_{i}\left(\prod_{l=1}^{n} \mu_{F_{i l}}\left(x_{l}\right)\right)}{\sum_{i=1}^{m}\left(\prod_{l=1}^{n} \mu_{F_{i l}}\left(x_{l}\right)\right)}
$$

where $\mu_{F_{i l}}\left(x_{l}\right)$ is the membership of $x_{1}$ in the fuzzy set $F_{i 1}$ and $\bar{y}_{i}=\max _{y \in R} \mu_{G_{i}}\left(\bar{y}_{i}\right)=1$. Then, (2.3) can be rewritten as

$$
\bar{f}\left(\bar{x}_{n}\right)=\theta_{f}^{T} \varphi_{f}\left(\bar{x}_{n}\right),
$$

where $\theta_{f}=\left[\bar{y}_{1}, \bar{y}_{2}, \ldots, \bar{y}_{m}\right]^{T}$ and $\varphi_{f}\left(\bar{x}_{n}(t)\right)=\left[\varphi_{11}, \ldots, \varphi_{1 m}\right]^{T}$. We can define $\varphi_{1 i}$ as

$$
\varphi_{1 i}=\frac{\left(\prod_{l=1}^{n} \mu_{F_{i l}}\left(x_{l}\right)\right)}{\sum_{i=1}^{m}\left(\prod_{l=1}^{n} \mu_{F_{i l}}\left(x_{l}\right)\right)}, \quad i=1, \ldots, m
$$

The logic fuzzy system shown in (2.5) is a universal approximator. It can be proved using the following lemma.

Lemma 2.1 (see [41]). Let $f\left(\bar{x}_{n}\right)$ be continuous functions defined on a compact set $U \in R^{n}$ and arbitrary $\varepsilon>0$, and there exists a fuzzy logic system $\bar{f}\left(\bar{x}_{n}\right)$ in the form of (2.4) such that

$$
\sup _{x \in U}\left|\bar{f}\left(\bar{x}_{n}\right)-f_{n}\left(\bar{x}_{n}\right)\right| \leq \varepsilon
$$

After some simple manipulations in (2.4) and (2.5), we can obtain $\bar{g}_{n}\left(\bar{x}_{n}\right)=\theta_{g}^{T} \varphi_{g}\left(\bar{x}_{n}\right)$ as the approximator of $g_{n}\left(\bar{x}_{n}\right)$. The nonlinear functions $f_{n}\left(\bar{x}_{n}\right)$ and $g_{n}\left(\bar{x}_{n}\right)$ requires successful estimates $\hat{\theta}_{f}$ and $\hat{\theta}_{g}$ in order to perform the performance shown in (2.6). 
Typically, there exists optimal parameter estimates $\bar{\theta}$, and the approximation error is the smallest. The optimal parameter estimate is defined as

$$
\begin{aligned}
& \bar{\theta}_{f}=\arg \min _{\theta_{f} \in \Omega_{f}}\left\{\sup _{\bar{x} \in \Omega_{\bar{x}}}\left|f_{n}\left(\bar{x}_{n}\right)-\theta_{f}^{T} \varphi_{f}\right|\right\}, \\
& \bar{\theta}_{g}=\arg \min _{\theta_{g} \in \Omega_{g}}\left\{\sup _{\bar{x} \in \Omega_{\bar{x}}}\left|g_{n}\left(\bar{x}_{n}\right)-\theta_{g}^{T} \varphi_{g}\right|\right\} .
\end{aligned}
$$

Based on fuzzy mechanism, (2.1) can be rewritten as below:

$$
\begin{aligned}
\dot{x}_{1} & =x_{2}+d_{1}(t), \\
\dot{x}_{2} & =x_{3}+d_{2}(t), \\
& \vdots \\
\dot{x}_{n} & =\bar{\theta}_{f}^{T} \varphi_{f}\left(\bar{x}_{n}\right)+\bar{\theta}_{g}^{T} \varphi_{g}\left(\bar{x}_{n}\right) u(t)+d_{n}(t)+\varepsilon_{f}+\varepsilon_{g}, \\
y & =x_{1},
\end{aligned}
$$

where $\varepsilon_{f}$ and $\varepsilon_{g}$ are internal modeling error variables

$$
\begin{gathered}
\varepsilon_{f}=f_{n}\left(\bar{x}_{n}\right)-\bar{\theta}_{f}^{T} \varphi_{f}\left(\bar{x}_{n}\right), \\
\varepsilon_{g}=g_{n}\left(\bar{x}_{n}\right)-\bar{\theta}_{g}^{T} \varphi_{g}\left(\bar{x}_{n}\right),
\end{gathered}
$$

Therefore, the mathematical model includes internal modeling error variables and external disturbance. We will discuss our proposed method in the next section.

\section{Adaptive Backstepping Fuzzy Controller Technique and Fuzzy-Immune Mechanism}

$f_{n}\left(\bar{x}_{n}(t)\right)$ and $g_{n}\left(\bar{x}_{n}(t)\right)$ are the system dynamic functions, these cannot be exactly obtained in general, $d_{1}(t), \ldots, d_{n}(t)$ are unknown parameters in practical application Thus, in the adaptive backstepping fuzzy controller (ABFC) system, the fuzzy system is designed to estimate the system dynamic functions.

\subsection{Backstepping Design Principle}

The design of ABFC for the chaotic dynamic system is described step-by-step as follows:

Step 1. Consider the tracking error

$$
\dot{e}_{1}=\dot{x}_{1}-\dot{r}=x_{2}-\dot{r}+d_{1}=e_{2}+a_{1}+d_{1}
$$


where $r$ is the command trajectory. The first Lyapunov function is defined as

$$
V_{1}=\frac{e_{1}^{2}}{2}
$$

Differentiating (3.2) with respect to time and it is obtained that

$$
\dot{V}_{1}=e_{1} \dot{e}_{1}=e_{1}\left(e_{2}+a_{1}+d_{1}\right)=e_{1} e_{2}+e_{1}\left(a_{1}+d_{1}\right) .
$$

Define the following stabilizing function:

$$
a_{1}=-\tau_{1} e_{1}-\frac{1}{\eta_{1}^{2}} e_{1}
$$

where $\tau_{1}$ and $\eta_{1}$ are positive constants, $\eta_{1}$ represent the attenuation level of disturbances. From substitute (3.3) into (3.4), then we obtain

$$
\dot{V}_{1}=-\tau_{1} e_{1}^{2}+e_{1} e_{2}+\mu_{1}
$$

where $\mu_{1}=-\left(1 / \eta_{1}^{2}\right) e_{1}+e_{1} d_{1}=-\left(\left(1 / \eta_{1}\right) e_{1}-(1 / 2) \eta_{1} d_{1}\right)^{2}+(1 / 4) \eta_{1}^{2} d_{1}^{2}$.

Step 2. The derivative of $e_{2}=x_{2}-\dot{r}-a_{1}$ is

$$
\dot{e}_{2}=\dot{x}_{3}+d_{2}-\ddot{r}-\frac{\partial a_{1}\left(x_{2}+d_{1}\right)}{\partial x_{1}}-\frac{\partial a_{1} \dot{r}}{\partial r_{1}}=e_{3}+a_{2}-\frac{\partial a_{1} x_{2}}{\partial x_{1}}-\frac{\partial a_{1} \dot{r}}{\partial r_{1}}+\left(d_{2}-\frac{\partial a_{1} d_{1}}{\partial x_{1}}\right) .
$$

The second Lyapunov function is defined as

$$
V_{2}=V_{1}+\frac{e_{2}^{2}}{2}
$$

Differentiating (3.7) with respect to time, it is obtained that

$$
\dot{V}_{2}=e_{2} e_{3}-\tau_{1} e_{1}^{2}+e_{2}\left(e_{1}+a_{2}-\frac{\partial a_{1} x_{2}}{\partial x_{1}}-\frac{\partial a_{1} \dot{r}}{\partial r_{1}}\right)+\mu_{1}+e_{2} d_{2}-e_{2} \frac{\partial a_{1} d_{1}}{\partial x_{1}} .
$$

Define the following stabilizing function:

$$
a_{2}=-e_{1}-\tau_{2} e_{2}-\frac{1}{\eta_{2}^{2}} e_{2}+\frac{\partial a_{1}}{\partial x_{1}} x_{2}+\frac{\partial a_{1}}{\partial r} \dot{r}
$$

where $\tau_{2}$ and $\eta_{2}$ are positive constants and $\eta_{2}$ represent the attenuation level of disturbances. From substitute (3.9) into (3.8), then we obtain

$$
\dot{V}_{2}=-\tau_{1} e_{1}^{2}-\tau_{2} e_{2}^{2}+e_{2} e_{3}+\mu_{1}+\mu_{2}
$$


where

$$
\mu_{2}=-\frac{1}{\eta_{2}^{2}} e_{2}^{2}+e_{2}\left(d_{2}-\frac{\partial a_{1}}{\partial x_{1}} d_{1}\right)=-\left[\frac{1}{\eta_{2}} e_{2}-\frac{1}{2} \eta_{2}\left(d_{2}-\frac{\partial a_{1}}{\partial x_{1}} d_{1}\right)\right]^{2}+\frac{1}{4} \eta_{2}^{2}\left(d_{2}-\frac{\partial a_{1}}{\partial x_{1}} d_{1}\right)^{2}
$$

Step $i$

After some simple manipulations and the same method, the derivative of generalized error is

$$
\dot{e}_{i}=x_{i+1}+\left[d_{i}-\sum_{l=1}^{i-1}\left(\frac{\partial a_{i-1}}{\partial x_{l}}\right) d_{l}\right]-\frac{d^{i} r}{d r}-\left(\sum_{l=1}^{i-1} \frac{\partial a_{i-1} x_{l+1}}{\partial x_{l}}+\sum_{l=1}^{i-1} \frac{\partial a_{i-1}}{\partial r^{(l-1)}} r^{l}\right)
$$

The ith Lyapunov function is defined as

$$
V_{i}=V_{i-1}+\frac{e_{i}^{2}}{2}
$$

Differentiating (3.13) with respect to time, it is obtained that

$$
\dot{V}_{i}=e_{i} e_{i+1}-\sum_{l=1}^{i} \tau_{l} e_{l}^{2}+\sum_{l=1}^{i} \eta_{l}
$$

where

$$
\mu_{l}=-\left[\frac{1}{\eta_{l}} e_{l}-\frac{1}{2} \eta_{l}\left(d_{l}-\sum_{k=1}^{l-1} \frac{\partial a_{l-1}}{\partial x_{k}} d_{k}\right)\right]^{2}+\frac{1}{4} \eta_{l}^{2}\left(d_{l}-\sum_{k=1}^{l-1} \frac{\partial a_{l-1}}{\partial x_{k}} d_{k}\right)^{2} .
$$

Define the following stabilizing function:

$$
a_{i}=-e_{i-1}-\tau_{i} e_{i}-\frac{1}{\eta_{i}^{2}} e_{i}+\sum_{l=1}^{i-1} \frac{\partial a_{i-1}}{\partial x_{l}} x_{l+1}+\sum_{l=1}^{i-1} \frac{\partial a_{i-1}}{\partial r^{(l-1)}} r^{l}
$$


Step $n$

The final control law will be determined in the final step, and the derivative of step generalized error can be described as

$$
\begin{aligned}
\dot{e}_{n}= & \dot{x}_{n}-\frac{d^{n} r}{d r}-\left(\sum_{l=1}^{n-1} \frac{\partial a_{n-1} x_{l+1}}{\partial x_{l}}+\sum_{l=1}^{n-1} \frac{\partial a_{n-1}}{\partial r^{(l-1)}} r^{l}\right) \\
= & \bar{\theta}_{f}^{T} \varphi_{f}\left(\bar{x}_{n}\right)+\bar{\theta}_{g}^{T} \varphi_{g}\left(\bar{x}_{n}\right) u-\frac{d^{n} r}{d r}-\left(\sum_{l=1}^{n-1} \frac{\partial a_{n-1} x_{l+1}}{\partial x_{l}}+\sum_{l=1}^{n-1} \frac{\partial a_{n-1}}{\partial r^{(l-1)}} r^{l}\right) \\
& +d_{n}-\sum_{l=1}^{n-1}\left(\frac{\partial a_{n-1}}{\partial x_{l}}\right) d_{l}+\varepsilon_{f}+\varepsilon_{g} u, \\
u= & \frac{1}{\hat{\theta}_{g}^{T} \varphi_{g}}\left(a_{n}+\frac{d^{n} r}{d r}\right), \quad \widehat{\theta}_{g}^{T} \varphi_{g} \neq 0, \\
a_{n}= & -e_{n-1}-\tau_{n} e_{n}-\frac{1}{\eta_{n}^{2}} e_{n}-\widehat{\theta}_{f}^{T} \varphi_{f}\left(\bar{x}_{n}\right)+\sum_{l=1}^{n-1} \frac{\partial a_{n-1}}{\partial x_{l}} x_{l+1}+\sum_{l=1}^{n-1} \frac{\partial a_{n-1}}{\partial r^{l-1}} r^{l},
\end{aligned}
$$

where $\eta_{n}$ is the given correct factor and $\tau_{n}$ is a positive constant.

\subsection{Fuzzy-Immune Mechanism Design}

Assume that the number of the $k$ th-generation antigens is $\varepsilon(k)$, the output of the helper Tcells, stimulated by antigens, is $T_{H}(k)$, and the suppressor T-cells affect B-cells to the amount of $T_{s}(k)[42,43]$

$$
T_{e}(k)=T_{H}(k)-T_{s}(k),
$$

where $T_{H}(k)=h_{1} \varepsilon(k)$ and $T_{s}(k)=h_{2} f\left(T_{e}(k), \Delta T_{e}(k)\right) \varepsilon(k)$. The feedback control rules are defined as

$$
\begin{aligned}
u(k) & =h_{1} e(k)-h_{2} f(u(k), \Delta u(k)) e(k) \\
& =h_{1}\left[1-\frac{h_{2}}{h_{1}} f(u(k), \Delta u(k))\right] e(k) \\
& =h_{1}[1-\hbar f(u(k), \Delta u(k))] e(k),
\end{aligned}
$$

where $h_{1}, h_{2}$, and $\lambda$ are scaling factors and $\hbar=h_{2} / h_{1}$ is utilized to control the stabilization effect. Then, we propose a novel correct constriction coefficient to improve the performance of the immune mechanism. In this, the constriction coefficient can be expressed as follows:

$$
\hbar=\hbar_{\max }-\frac{\hbar_{\max }-\hbar_{\min }}{k_{\max }} \times k_{\text {now }}
$$




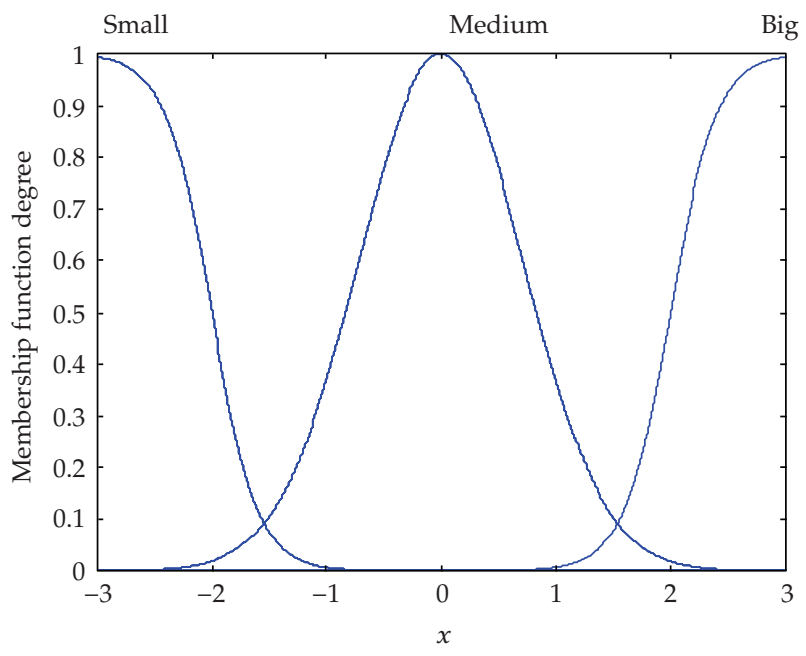

Figure 1: Membership functions for $u(k)$.

where $\hbar_{\max }$ and $\hbar_{\min }$ denote the maximum and minimum of $\hbar$, respectively. $k_{\max }$ denotes the total number of evolution generations, and $k_{\text {now }}$ denotes the current number of evolution generations. Let de $(k)$ denote the difference between the $e(k)$ 's for two consecutive iterations, that is, at iteration $k$ :

$$
\operatorname{de}(k)=e(k)-e(k-1) \geq 0 .
$$

Based on the above inferences, the following nine fuzzy rules are suggested.

(1) If $u(k)$ is big and $\Delta u(k)$ is big, then $f(u(k), \Delta u(k))$ is small.

(2) If $u(k)$ is big and $\Delta u(k)$ is small, then $f(u(k), \Delta u(k))$ is medium.

(3) If $u(k)$ is small and $\Delta u(k)$ is big, then $f(u(k), \Delta u(k))$ is medium.

(4) If $u(k)$ is medium and $\Delta u(k)$ is big, then $f(u(k), \Delta u(k))$ is small.

(5) If $u(k)$ is big and $\Delta u(k)$ is medium, then $f(u(k), \Delta u(k))$ is small.

(6) If $u(k)$ is medium and $\Delta u(k)$ is medium, then $f(u(k), \Delta u(k))$ is medium.

(7) If $u(k)$ is medium and $\Delta u(k)$ is small, then $f(u(k), \Delta u(k))$ is medium.

(8) If $u(k)$ is small and $\Delta u(k)$ is medium, then $f(u(k), \Delta u(k))$ is big.

(9) If $u(k)$ is small and $\Delta u(k)$ is small, then $f(u(k), \Delta u(k))$ is big.

The membership functions for $u(k), \Delta u(k)$, and $f(u(k), \Delta u(k))$ are shown in Figures $1-3$, respectively.

The output of the controller has the following expression:

$$
u=\frac{1}{\widehat{\theta}_{g}^{T} \varphi_{g}}\left(a_{n}+\frac{d^{n} r}{d r}\right)\left\{h_{1}[1-\hbar f(u(k), \Delta u(k))] e_{n}\right\}
$$

The proposed method is trying to find more efficient ways of utilizing immune mechanism to correct controller input, and the correct item is effective in keeping out 


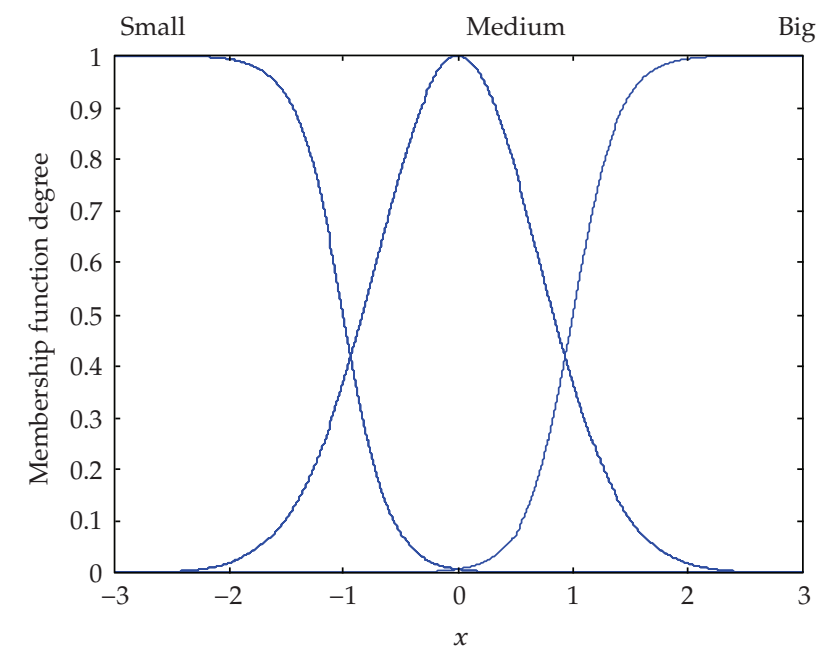

Figure 2: Membership functions for $\Delta u(k)$.

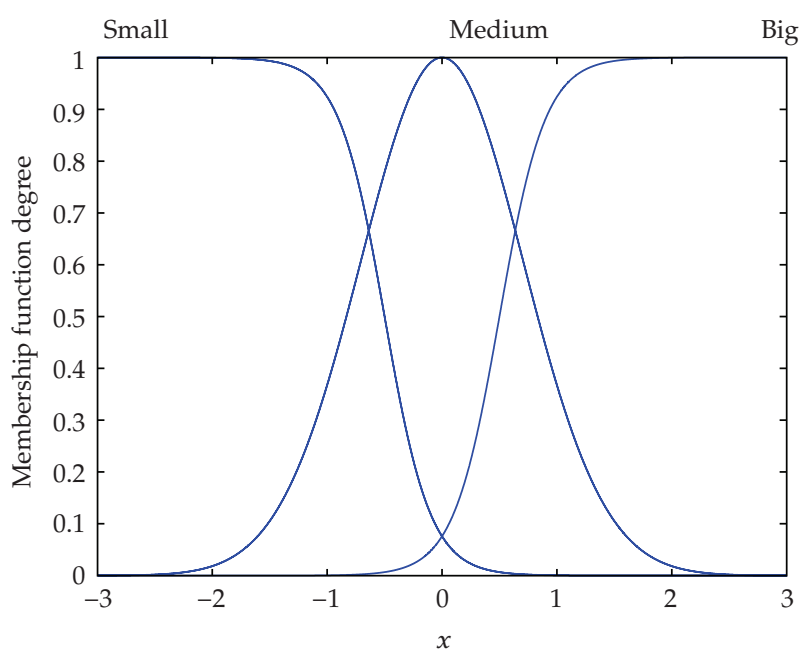

Figure 3: Membership functions for $f(u(k), \Delta u(k))$.

perturbation by external or internal. Then, we have the following theorem to gain the objective.

Theorem 3.1. Consider the nonlinear systems (2.1) and with the controller $u$ is given by (3.18). By utilizing parameter adjusting law (3.25) and (3.26)

$$
\begin{aligned}
& \dot{\hat{\theta}}_{f}=P^{-1} \varphi_{f}\left(\bar{x}_{n}\right) e_{n}, \\
& \dot{\hat{\theta}}_{g}=Q^{-1} \varphi_{g}\left(\bar{x}_{n}\right) e_{n} u,
\end{aligned}
$$


then the proposed adaptive backstepping fuzzy-immune control scheme can guarantee the following properties:

(1) The asymptotic stability of the system is guaranteed.

(2) The tracking error can be described as

$$
\sum_{l=1}^{n} \int_{0}^{T}\left(-\tau_{l} e_{l}^{2}\right) d t \leq V(0)+\sum_{l=1}^{n} \int_{0}^{T}\left(\frac{1}{4} \eta_{l}^{2}\left(d_{l}-\sum_{k=1}^{l-1} \frac{\partial a_{l-1}}{\partial x_{k}} d_{k}\right)^{2}\right) d t
$$

Proof. Firstly, consideration of fuzzy approximating error, the Lyapunov functional is set as (3.28)

$$
V=V_{n-1}+\frac{1}{2} e_{n}^{2}+\frac{1}{2} \tilde{\theta}_{f}^{T} P \tilde{\theta}_{f}+\frac{1}{2} \tilde{\theta}_{g}^{T} Q \tilde{\theta}_{g}
$$

where $P$ and $Q$ are positive symmetric matrices.

Differentiating (3.28) with respect to time, it is obtained that

$$
\begin{aligned}
\dot{V}= & \dot{V}_{n-1}+e_{n} \dot{e}_{n}+\tilde{\theta}_{f}^{T} P^{-1} \dot{\tilde{\theta}}_{f}+\tilde{\theta}_{g}^{T} Q^{-1} \dot{\tilde{\theta}}_{g} \\
= & e_{n-1} e_{n}+e_{n}\left(\hat{\theta}_{g}^{T} \varphi_{g}\left(\bar{x}_{n}\right) u-\frac{d^{n} r}{d r}-a_{n}\right)+\sum_{l=1}^{n-1}\left(\mu_{l}-\tau_{l} e_{l}^{2}\right) \\
& +\tilde{\theta}_{f}^{T} P\left(P^{-1} \varphi_{f}\left(\bar{x}_{n}\right) e_{n}-\dot{\hat{\theta}}_{f}\right)+\tilde{\theta}_{g}^{T} Q\left(Q^{-1} \varphi_{g}\left(\bar{x}_{n}\right) e_{n} u-\dot{\hat{\theta}}_{f}\right) \\
& +e_{n}\left(\left(\bar{\theta}_{f}^{T} \varphi_{f}\left(\bar{x}_{n}\right)+a_{n}-\left(\sum_{l=1}^{n-1} \frac{\partial a_{n-1} x_{l+1}}{\partial x_{l}}+\sum_{l=1}^{n-1} \frac{\partial a_{n-1}}{\partial r^{(l-1)}} r^{l}+d_{n}-\sum_{l=1}^{n-1}\left(\frac{\partial a_{n-1}}{\partial x_{l}}\right) d_{l}+\varepsilon_{f}+\varepsilon_{g} u\right)\right)\right) .
\end{aligned}
$$

Define

$$
\dot{V}_{n}=\dot{V}_{n-1}+e_{n}\left\{\bar{\theta}_{f}^{T} \varphi_{f}\left(\bar{x}_{n}\right)+a_{n}-\left(\sum_{l=1}^{n-1} \frac{\partial a_{n-1} x_{l+1}}{\partial x_{l}}+\sum_{l=1}^{n-1} \frac{\partial a_{n-1}}{\partial r^{(l-1)}} r^{l}+d_{n}-\sum_{l=1}^{n-1}\left(\frac{\partial a_{n-1}}{\partial x_{l}}\right) d_{l}+\varepsilon_{f}+\varepsilon_{g} u\right)\right\} .
$$

Equation (3.29) can be rewritten as

$$
\dot{V}=\dot{V}_{n}+e_{n}\left(\widehat{\theta}_{g}^{T} \varphi_{g}\left(\bar{x}_{n}\right) u-\frac{d^{n} r}{d r}-a_{n}\right)+\tilde{\theta}_{f}^{T} P\left(P^{-1} \varphi_{f}\left(\bar{x}_{n}\right) e_{n}-\dot{\hat{\theta}}_{f}\right)+\tilde{\theta}_{g}^{T} Q\left(Q^{-1} \varphi_{g}\left(\bar{x}_{n}\right) e_{n} u-\dot{\hat{\theta}}_{g}\right)
$$

Define $\dot{V}=-\sum_{l=1}^{n} \tau_{l} e_{l}^{2}+\sum_{l=1}^{n} \mu_{l}$. 
By using (3.18) and adaptive laws (3.25)-(3.26), (3.31) can be written as

$\dot{V}=-\sum_{l=1}^{n} \tau_{l} e_{l}^{2}+\sum_{l=1}^{n} \mu_{l}=\sum_{l=1}^{n}\left\{-\left[\frac{1}{\eta_{l}} e_{l}-\frac{1}{2} \eta_{l}\left(d_{l}-\sum_{k=1}^{l-1} \frac{\partial a_{l-1}}{\partial x_{k}} d_{k}\right)\right]^{2}+\frac{1}{4} \eta_{l}^{2}\left(d_{l}-\sum_{k=1}^{l-1} \frac{\partial a_{l-1}}{\partial x_{k}} d_{k}\right)^{2}\right\}-\sum_{l=1}^{n} \tau_{l} e_{l}^{2}$.

Integrating (3.32) from $t=0$ to $T$ yields

$$
V(T)-V(0) \leq \sum_{l=1}^{n} \int_{0}^{T}\left(-\tau_{l} e_{l}^{2}+\frac{1}{4} \eta_{l}^{2}\left(d_{l}-\sum_{k=1}^{l-1} \frac{\partial a_{l-1}}{\partial x_{k}} d_{k}\right)^{2}\right) d t
$$

Furthermore, one can derive that

$$
\sum_{l=1}^{n} \int_{0}^{T}\left(-\tau_{l} e_{l}^{2}\right) d t \leq V(0)+\sum_{l=1}^{n} \int_{0}^{T}\left(\frac{1}{4} \eta_{l}^{2}\left(d_{l}-\sum_{k=1}^{l-1} \frac{\partial a_{l-1}}{\partial x_{k}} d_{k}\right)^{2}\right) d t
$$

This completes the proof of the theorem.

\section{Illustrative Examples}

In this section, two examples are provided to illustrate the usefulness of our method.

Example 4.1. Consider the following Duffing-Holmes chaotic system [40]:

$$
\begin{gathered}
\dot{x}_{1}=x_{2}+d_{1}(t), \quad d_{1}(t)=0.1 \sin t+0.2 \cos t, \\
\dot{x}_{2}=-a x_{2}-b x_{1}^{3}-c x_{1}+q \cos (\omega t)+\left(1+\cos x_{1}\right) u+d_{2}(t), \quad d_{2}(t)=0.1 \cos t,
\end{gathered}
$$

where $a, b, c$ and $q$ are constants, $\omega$ is the frequency, $d_{1}(t)$ and $d_{2}(t)$ are external disturbance, and $u$ is the control effort. For observing chaotic unpredictable behavior, the open-loop system behavior with $u=0, d_{1}(t)=d_{2}(t)=0$ was simulated with $a=0.1, b=1.0, c=1, q=12$, and $\omega=1.0$. The phase plane plots from an initial condition point $(0,0)$ are shown in Figure 4 . The proposed controller is designed to force the system output to track the given desired trajectory $y_{m}=\sin t$. Now, choose $y=x_{1}$, and there exist external disturbances when the chaotic systems have the form of strict-feedback. It is assumed that the external disturbance $d_{1}(t)=0.1 \sin t+0.2 \cos t$ and $d_{2}(t)=0.1 \cos t$. By Lemma 2.1, we use using some fuzzy rules 


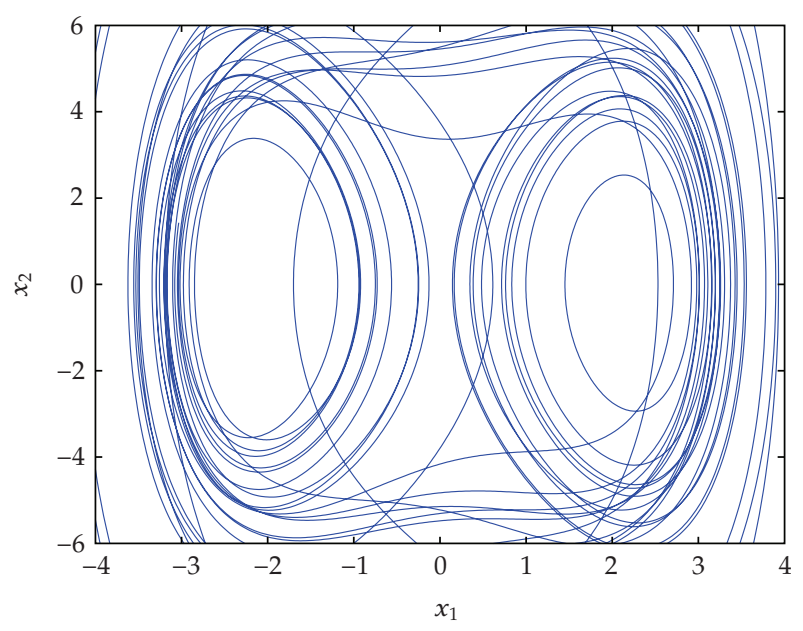

Figure 4: State trajectories of the Duffing equation.

for approximation of the function $f_{n}\left(\bar{x}_{n}(t)\right)$ and $g_{n}\left(\bar{x}_{n}(t)\right)$. The membership functions of the fuzzy sets are expressed as

$$
\begin{aligned}
& \mu_{F_{i}^{1}}\left(x_{i}\right)=\frac{1}{1+\exp \left(5 \times\left(x_{i}+2\right)\right)} \\
& \mu_{F_{i}^{2}}\left(x_{i}\right)=\exp \left[-\left(x_{i}+1\right)^{2}\right] \\
& \mu_{F_{i}^{3}}\left(x_{i}\right)=\exp \left[-x_{\mathrm{i}}^{2}\right] \\
& \mu_{F_{i}^{4}}\left(x_{i}\right)=\exp \left[-\left(x_{i}-1\right)^{2}\right] \\
& \mu_{F_{i}^{5}}\left(x_{i}\right)=\frac{1}{1+\exp \left(5 \times\left(x_{i}-2\right)\right)}
\end{aligned}
$$

The initial membership functions for $u(k), \Delta u(k)$, and $f(u(k), \Delta u(k))$ are shown in Figures 1-3, respectively. In order to evaluate the performance of the adaptive backstepping fuzzy-immune control applied to the above system (4.1), it was compared to fuzzy adaptive control (FAC) [40]. Figures 5 and 6 show the simulation curves of the two controls for $x_{1}$ and $x_{2}$, for the system described by (4.1) under the initial conditions $x(0)=[2,2]$ with external disturbance $d_{1}(t)=0.1 \sin t+0.2 \cos t$ and $d_{2}(t)=0.1 \cos t$. In order to compare the stabilization and tracking performance, we consider the example introduced in [40]. FAC and the proposed method require 3.5 4.0 seconds and 2.5 3.0 seconds to track the reference signal, respectively. The proposed scheme can also suppress system uncertainty and disturbance, and its ISE (integral square error criterion) is lower than FAC. Figures 7 and 8 show $x_{1}$ and $x_{2}$ for the nonlinear system described by (4.1) under initial states $[-1.5,-1.5]$, $[5,-2]$, and $[-5,2]$ with external disturbance $d_{1}(t)=0.1 \sin t+0.2 \cos t$ and $d_{2}(t)=0.1 \cos t$, respectively. Finally, all simulation results are given in Table 1. 


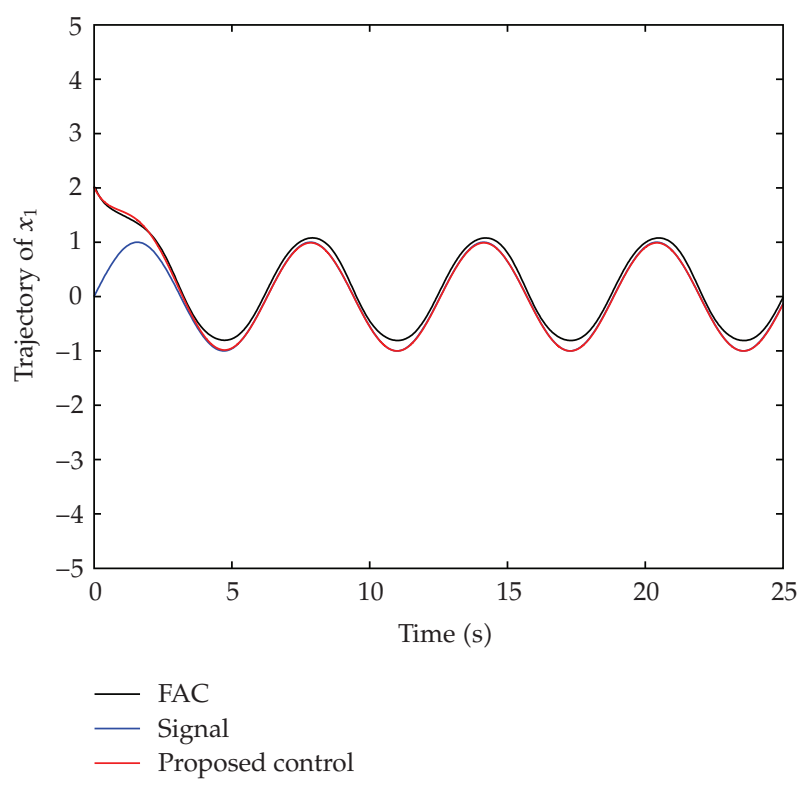

Figure 5: The tracking result for $x_{1}$.

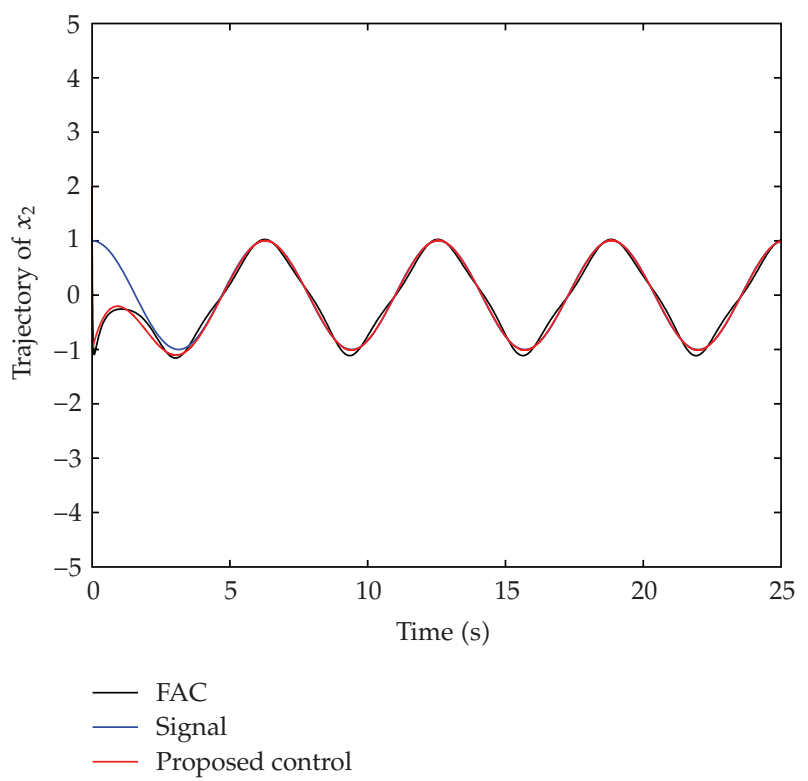

Figure 6: The tracking result for $x_{2}$.

Table 1: Experiments results for FAC and proposed control methods.

\begin{tabular}{lccc}
\hline & Time $(\mathrm{sec})$ & ISE & Performance \\
\hline FAC [40] & $3.5 \sim 4.0$ & 0.30 & Affected by noise \\
Proposed & $2.5 \sim 3.0$ & 0.05 & Rejects noise \\
\hline
\end{tabular}




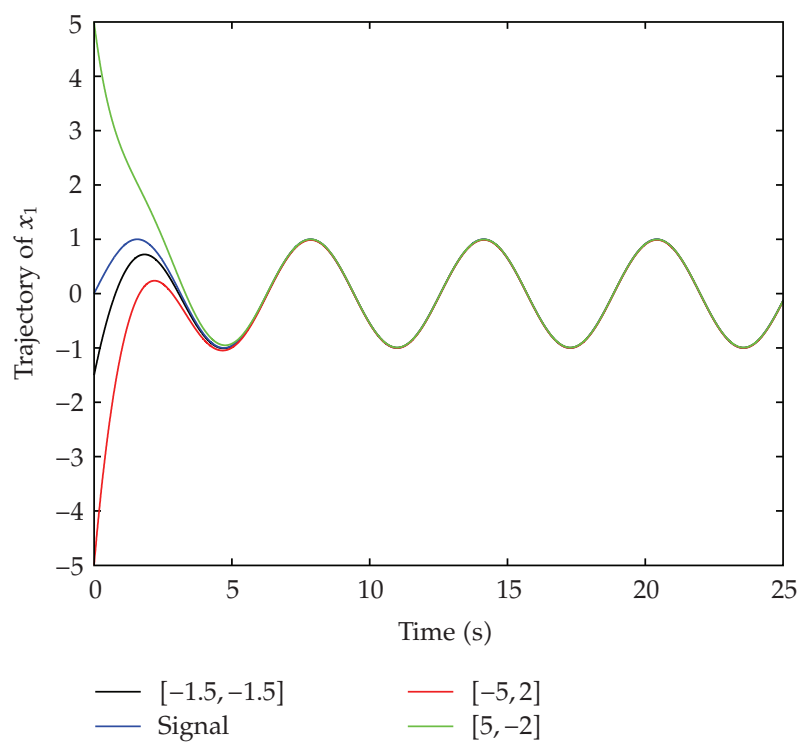

Figure 7: Trajectories of state $x_{1}$ under initial conditions.

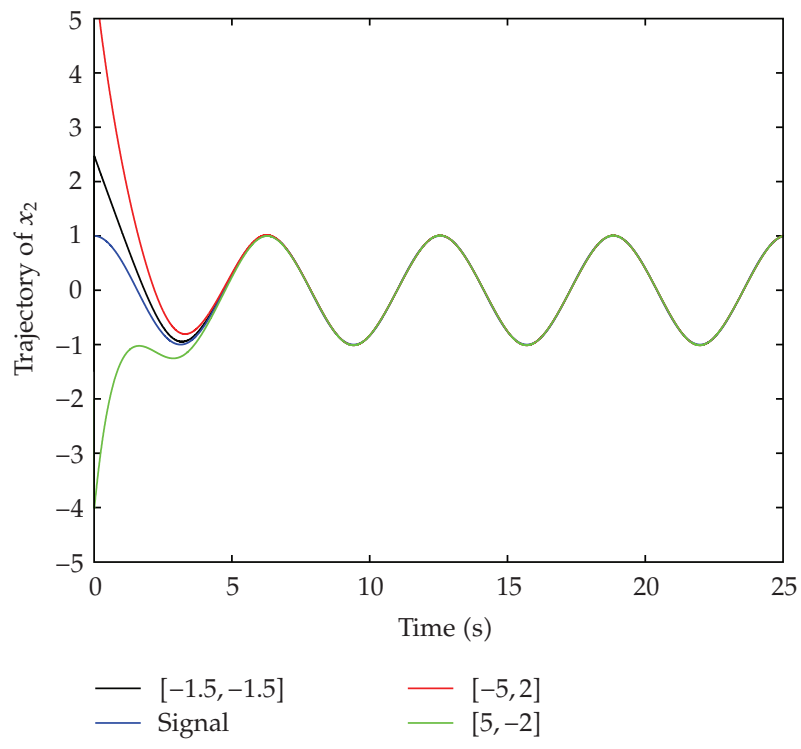

Figure 8: Trajectories of state $x_{2}$ under initial conditions.

Example 4.2. As a second example, consider the following forced chaotic attractor of the modified Chua circuit. The dynamics of the systems can be described as [44]

$$
\left[\begin{array}{l}
\dot{x}_{1} \\
\dot{x}_{2} \\
\dot{x}_{3}
\end{array}\right]=\left[\begin{array}{lll}
0 & 1 & 0 \\
0 & 0 & 1 \\
0 & 0 & 0
\end{array}\right]\left[\begin{array}{l}
x_{1} \\
x_{2} \\
x_{3}
\end{array}\right]+\left[\begin{array}{l}
0 \\
0 \\
1
\end{array}\right]\left[f+\left(1+\sin \left(x_{1}\right) u(t)\right)\right]+\left[\begin{array}{l}
d_{1}(t) \\
d_{2}(t) \\
d_{3}(t)
\end{array}\right] .
$$




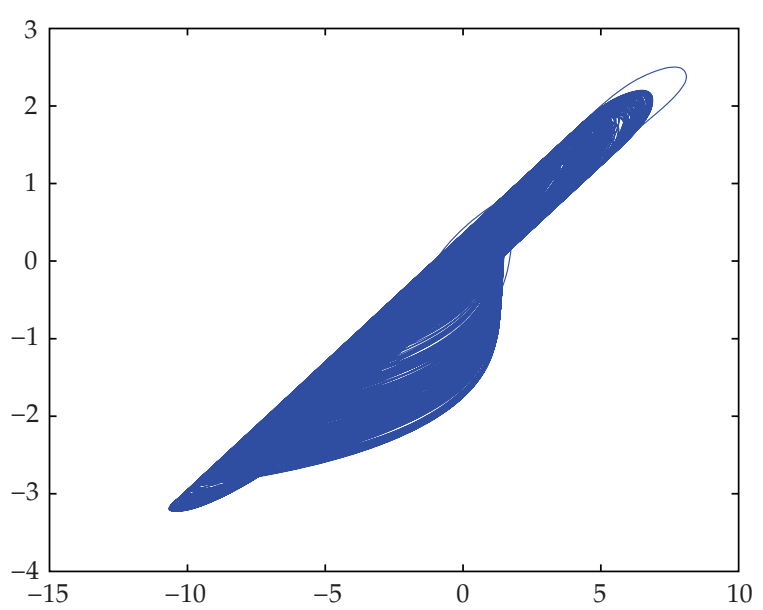

Figure 9: Chaotic attractor of the modified Chua circuit.

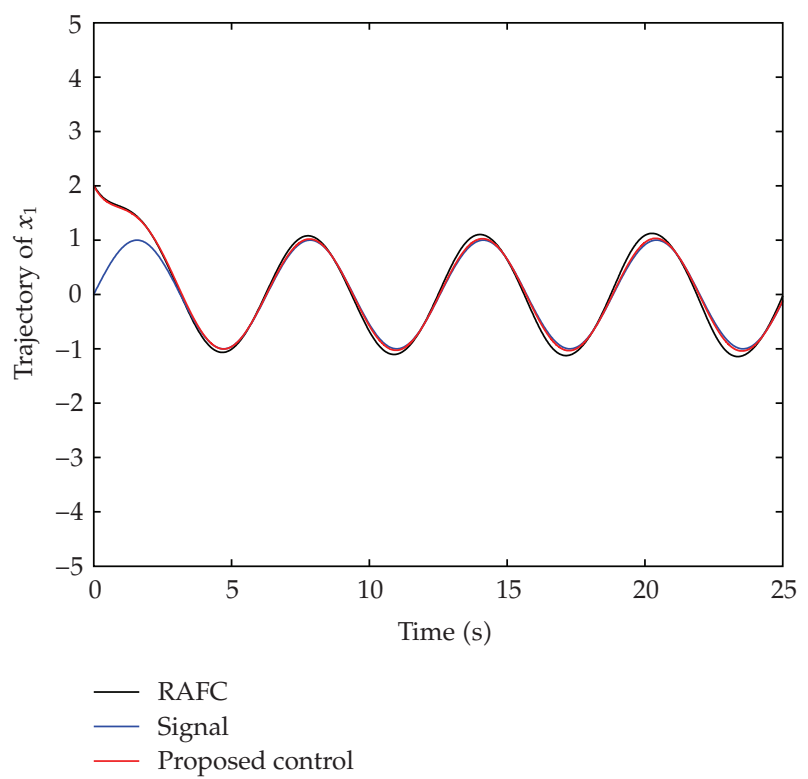

Figure 10: The tracking result for $x_{1}$.

In this simulation, let the sampling time equal 0.01 and the initial system states be $[2,1,3]$. Now, let $d_{1}(t), d_{2}(t)$ and $d_{3}(t)$ are external disturbance. If $d_{1}(t)=d_{2}(t)=d_{3}(t)=u(t)=$ 0 and $f=(14 / 1805) x_{1}-(168 / 9025) x_{2}+(1 / 38) x_{3}-(2 / 45) \times\left((28 / 321) x_{1}+(7 / 95) x_{2}+x_{3}\right)^{3}$, then the system (4.3) is chaotic system and the trajectories of the state variables $x_{1}, x_{2}$, and $x_{3}$ are shown in Figure 9. By Lemma 2.1, we use some fuzzy rules for approximation of the function $f_{n}\left(\bar{x}_{n}(t)\right)$ and $g_{n}\left(\bar{x}_{n}(t)\right)$. In order to control this chaotic system, the proposed control is utilized and the corresponding adaptation laws are also applied such that the system output $y$ tracks a desired trajectory. In addition, let the desired output be $y_{m}=\sin (t)$.

Simulated results are demonstrated in Figures 10, 11, 12, 13, 14 and 15. The initial membership functions for $u(k), \Delta u(k)$ and $f(u(k), \Delta u(k))$ are shown in Figures 1-3, 


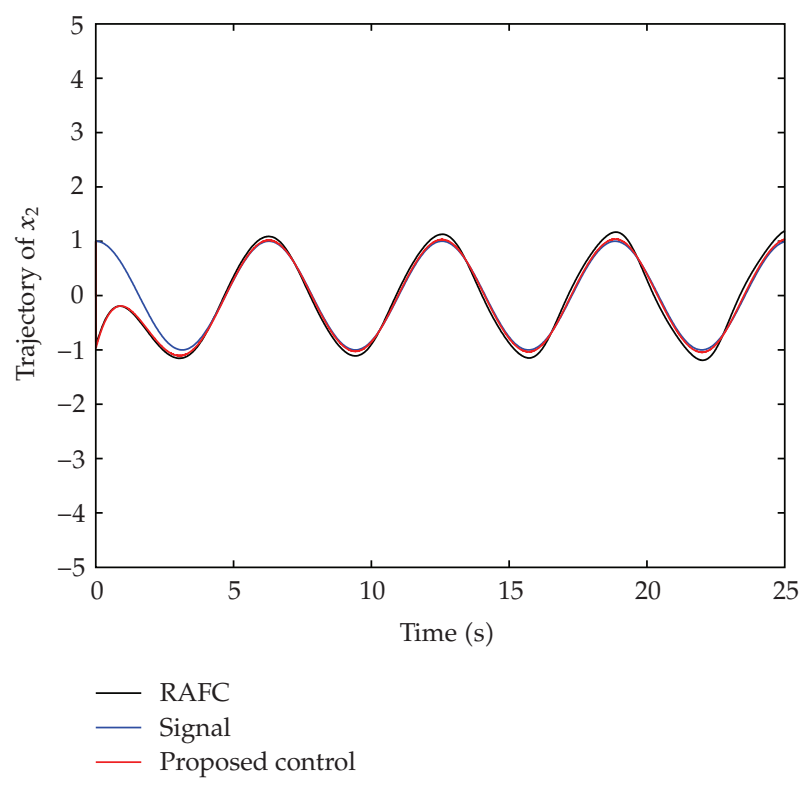

Figure 11: The tracking result for $x_{2}$.

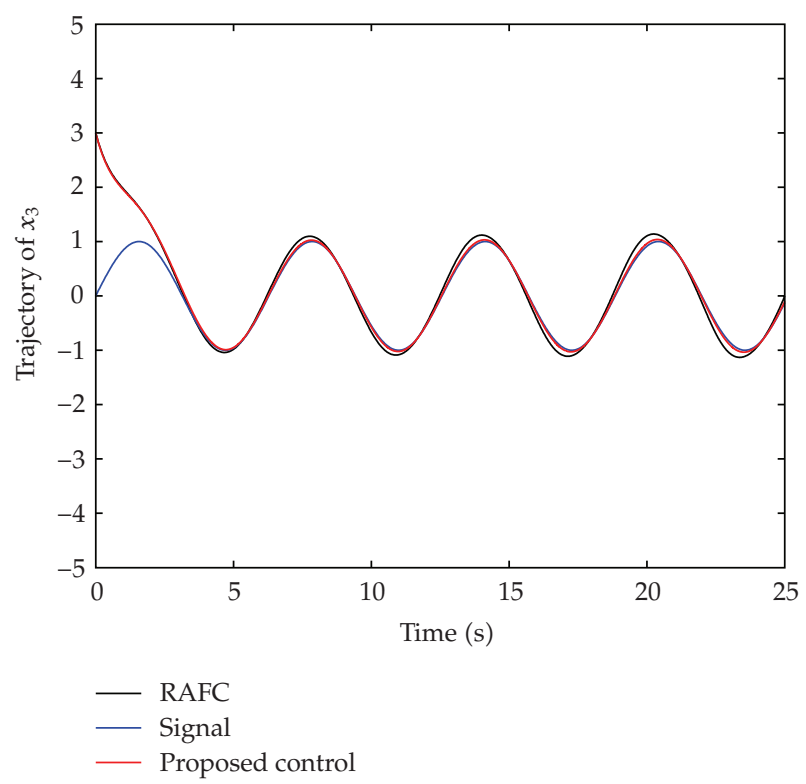

Figure 12: The tracking result for $x_{3}$.

respectively. In order to evaluate the performance of the adaptive backstepping fuzzyimmune control applied to the above system (4.3), it was compared to robust adaptive fuzzy controller (RAFC) [44]. Figures 10-12 show the simulation curves for the system described by (4.3) under the initial conditions $x(0)=[2,1,3]$ with external disturbance $d_{1}(t)=0.1 \cos t+0.4 \cos t, d_{2}(t)=0.2 \sin t$ and $d_{3}(t)=0.1 \sin t$. RFAC and the proposed method require 3.0 3.2 seconds and 2.5 3.0 seconds to track the reference signal, respectively. The 


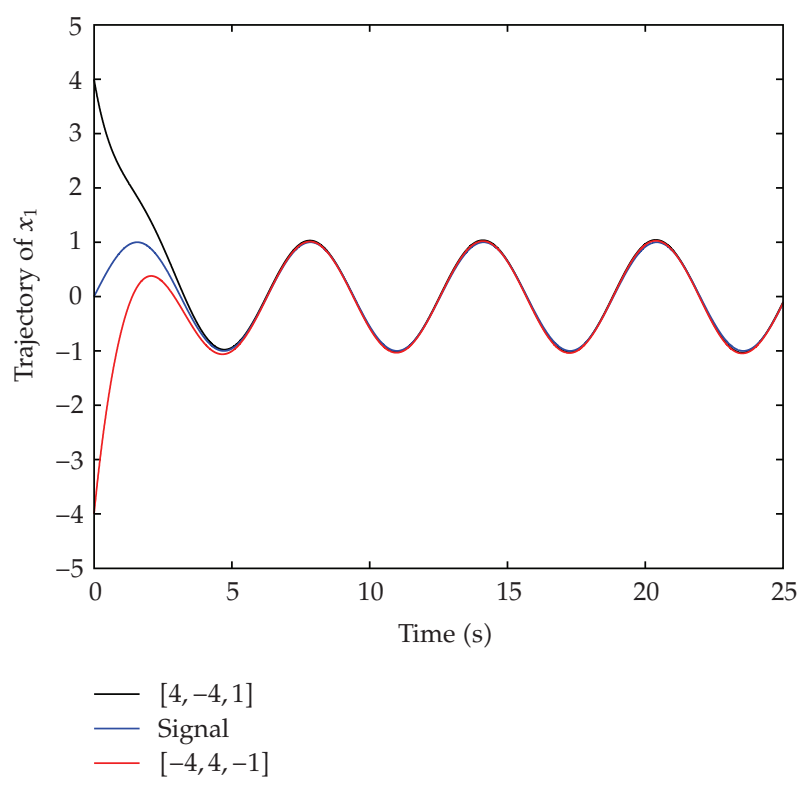

Figure 13: Trajectories of state $x_{1}$ under initial conditions.

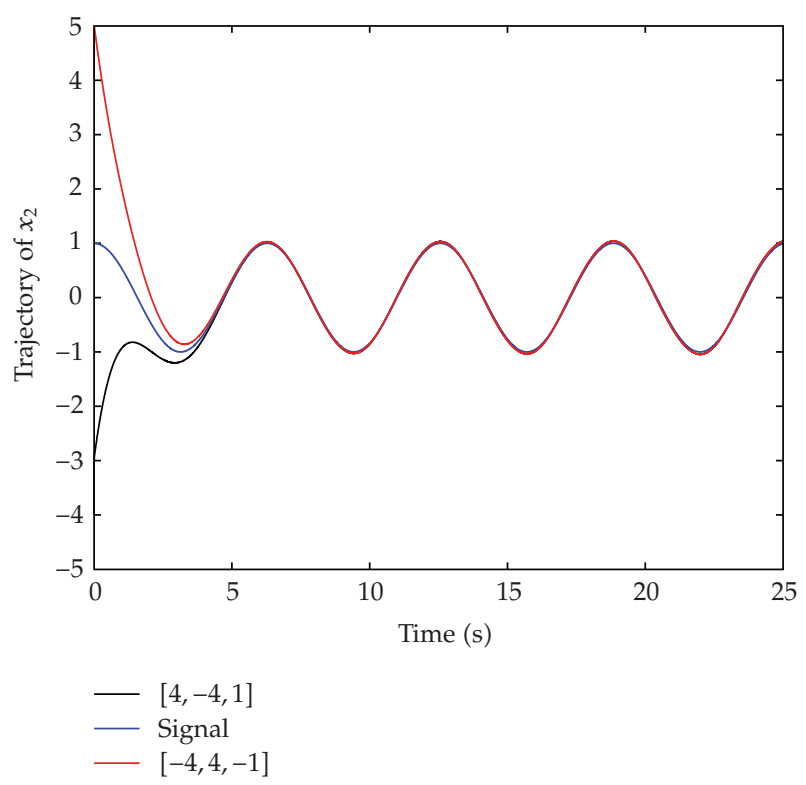

Figure 14: Trajectories of state $x_{2}$ under initial conditions.

proposed scheme can also suppress disturbance, and its ISE (integral square error criterion) is lower than RAFC. Figures 13-15 show the controlled stabilization of $x_{1}, x_{2}$, and $x_{3}$ for the nonlinear system described by (4.3) under initial states $[4,-4,1]$, and $[-4,4,-1]$ with external disturbance $d_{1}(t)=0.1 \cos t+0.4 \cos t, d_{2}(t)=0.2 \sin t$ and $d_{3}(t)=0.1 \sin t$. It seems to be satisfactory. Finally, all simulation results are given in Table 2. 


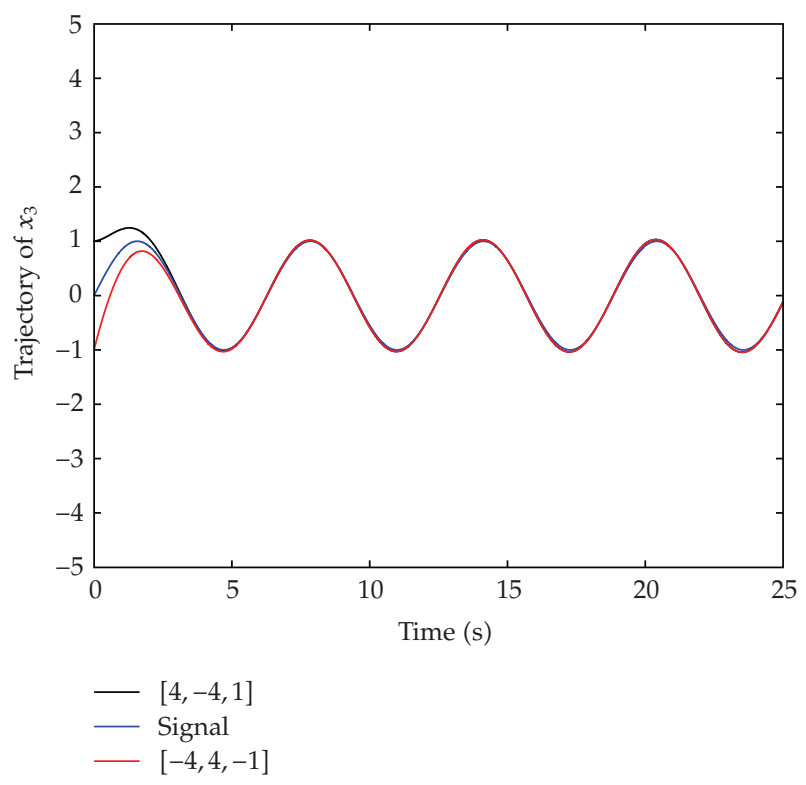

Figure 15: Trajectories of state $x_{3}$ under initial conditions.

Table 2: Experiments results for RAFC and proposed control methods.

\begin{tabular}{lccc}
\hline & Time $(\mathrm{sec})$ & ISE & Performance \\
\hline RAFC [44] & $3.0 \sim 3.2$ & 0.2 & Affected by noise \\
Proposed & $2.5 \sim 3.0$ & 0.05 & Rejects noise \\
\hline
\end{tabular}

\section{Conclusion}

A hybrid optimization algorithm that combines the adaptive backstepping principle and the fuzzy-immune algorithm for a class of chaotic dynamical systems was presented. The four main contributions of this paper are: (1) an adaptive backstepping fuzzy-immune tracking control method for a class of chaotic systems is designed, (2) the controller does not require a priori knowledge of the sign of the control coefficient, (3) fuzzy-immune algorithm is used to self-adjustment controller's coefficient for the optimal solution, and (4) a correct term is introduced to eliminate internal uncertainty and external disturbance. The proposed hybrid intelligence adaptive backstepping fuzzy-immune controller guarantees closed-loop stability while maitaining the desired tracking performance. Simulation results show that the proposed controller scheme provides better tracking performance than those of two existing methods.

\section{Acknowledgment}

This work was supported by the National Science Council of Taiwan, under Grant NSC-992221-E-027-101. 


\section{References}

[1] P. Angelov, “A fuzzy controller with evolving structure," Information Sciences, vol. 161, no. 1-2, pp. 21-35, 2004.

[2] C.-W. Chen, "Modeling and fuzzy PDC control and its application to an oscillatory TLP structure," Mathematical Problems in Engineering, vol. 2010, Article ID 120403, 13 pages, 2010.

[3] J. Llibre and M. A. Teixeira, "Limit cycles bifurcating from a two-dimensional isochronous cylinder," Applied Mathematics Letters, vol. 22, no. 8, pp. 1231-1234, 2009.

[4] P. C. Chen, C. W. Chen, and W. L. Chiang, "GA-based fuzzy sliding mode controller for nonlinear systems," Mathematical Problems in Engineering, vol. 2008, Article ID 325859, 16 pages, 2008.

[5] L. X. Wang, Adaptive Fuzzy Systems and Control: Design and Stability Analysis, Prentice Hall, Englewood Cliffs, NJ, USA, 1994.

[6] D. de Carvalho Braga, L. F. Mello, and M. Messias, "Bifurcation analysis of the van der Pol-Duffing circuit with parallel resistor," Mathematical Problems in Engineering, vol. 2009, Article ID 149563, 26 pages, 2009.

[7] J. M. V. Grzybowski, M. Rafikov, and E. E. N. MacAu, "Synchronization analysis for chaotic communication on a satellite formation flying," Acta Astronautica, vol. 67, no. 7-8, pp. 881-891, 2010.

[8] S. Ghaemi, S. Khanmohammadi, and M. Tinati, "Driver's behavior modeling using fuzzy logic," Mathematical Problems in Engineering, vol. 2010, Article ID 172878, 29 pages, 2010.

[9] J. Yu, J. Gao, Y. Ma, and H. Yu, "Adaptive fuzzy tracking control for a permanent magnet synchronous motor via backstepping approach," Mathematical Problems in Engineering, vol. 2010, Article ID 391846, 13 pages, 2010.

[10] M. de la Sen, "Adaptive stabilization of continuous-time systems through a controllable modified estimation model," Mathematical Problems in Engineering, no. 2, Article ID 2085987, pp. 109-131, 2004.

[11] F. L. Pereira and G. N. Silva, “Necessary conditions of optimality for vector-valued impulsive control problems," Systems E Control Letters, vol. 40, no. 3, pp. 205-215, 2000.

[12] L. F. R. Turci, E. E. N. Macau, and T. Yoneyama, "Efficient chaotic based satellite power supply subsystem," Chaos, Solitons and Fractals, vol. 42, no. 1, pp. 396-407, 2009.

[13] N. F. Al-Muthairi and M. Zribi, "Sliding mode control of a magnetic levitation system," Mathematical Problems in Engineering, vol. 2004, no. 2, pp. 93-107, 2004.

[14] V. A. de Oliveira, G. N. Silva, and M. A. Rojas-Medar, "KT-invexity in optimal control problems," Nonlinear Analysis. Theory, Methods E Applications, vol. 71, no. 10, pp. 4790-4797, 2009.

[15] M. de Pinho, "Mixed constrained control problems," Journal of Mathematical Analysis and Applications, vol. 278, no. 2, pp. 293-307, 2003.

[16] M. Corbera, J. Llibre, and M. A. Teixeira, "Symmetric periodic orbits near a heteroclinic loop in $R^{3}$ formed by two singular points, a semistable periodic orbit and their invariant manifolds," Physica D, vol. 238, no. 6, pp. 699-705, 2009.

[17] Y. Li and K. Zhao, "Robust stability analysis of fuzzy neural network with delays," Mathematical Problems in Engineering, vol. 2009, Article ID 826908, 13 pages, 2009.

[18] F. A. Breve, L. Zhao, M. G. Quiles, and E. E. N. Macau, "Chaotic phase synchronization and desynchronization in an oscillator network for object selection," Neural Networks, vol. 22, no. 5-6, pp. 728-737, 2009.

[19] M. de Pinho and J. F. Rosenblueth, "Necessary conditions for constrained problems under Mangasarian-Fromowitz conditions," SIAM Journal on Control and Optimization, vol. 47, no. 1, pp. 535-552, 2008.

[20] M. de Pinho and J. F. Rosenblueth, "Mixed constraints in optimal control: an implicit function theorem approach," IMA Journal of Mathematical Control and Information, vol. 24, no. 2, pp. 197-218, 2007.

[21] V. T. Minh and F. M. Hashim, "Adaptive teleoperation system with neural network-based multiple model control," Mathematical Problems in Engineering, vol. 2010, Article ID 592054, 15 pages, 2010.

[22] J. S. W. Lamb, M.-A. Teixeira, and K. N. Webster, "Heteroclinic bifurcations near Hopf-zero bifurcation in reversible vector fields in $R^{3}$," Journal of Differential Equations, vol. 219, no. 1, pp. 78-115, 2005.

[23] H. Peng, Y. Shao, L. Li, and Y. Yang, "Cryptanalysis of a chaotic communication scheme using parameter observer," Mathematical Problems in Engineering, vol. 2010, Article ID 361860, 18 pages, 2010.

[24] J.-N. Choi, S.-K. Oh, and W. Pedrycz, "Identification of fuzzy relation models using hierarchical fair competition-based parallel genetic algorithms and information granulation," Applied Mathematical Modelling, vol. 33, no. 6, pp. 2791-2807, 2009. 
[25] J. Lin and C. Fan, "Exponential admissibility and dynamic output feedback control of switched singular systems with interval time-varying delay," Mathematical Problems in Engineering, vol. 2010, Article ID 680382, 21 pages, 2010.

[26] J.-H. Park, G.-T. Park, S.-H. Kim, and C.-J. Moon, “Output-feedback control of uncertain nonlinear systems using a self-structuring adaptive fuzzy observer," Fuzzy Sets and Systems, vol. 151, no. 1, pp. 21-42, 2005.

[27] J.-Y. Cao, J.-P. Wen, and B.-G. Cao, “Robust ESO two-degree-of-freedom control design for permanent magnet synchronous motor," Mathematical Problems in Engineering, vol. 2010, Article ID 405251, 10 pages, 2010.

[28] J. Llibre, M. Messias, and P. R. da Silva, "On the global dynamics of the Rabinovich system," Journal of Physics, vol. 41, no. 27, p. 21, 2008.

[29] J. Baumeister, A. Leitão, and G. N. Silva, “On the value function for nonautonomous optimal control problems with infinite horizon," Systems E Control Letters, vol. 56, no. 3, pp. 188-196, 2007.

[30] S. Tong and Y. Li, "Direct adaptive fuzzy backstepping control for a class of nonlinear systems," International Journal of Innovative Computing, Information and Control, vol. 3, no. 4, pp. 887-896, 2007.

[31] X. Wei, M. F. Randrianandrasana, M. Ward, and D. Lowe, "Nonlinear dynamics of a periodically driven Duffing resonator coupled to a Van der Pol oscillator," Mathematical Problems in Engineering, vol. 2011, Article ID 248328, 16 pages, 2011.

[32] A. Elharfi, "Feedback controller stabilizing vibrations of a flexible cable related to an overhead crane," Mathematical Problems in Engineering, vol. 2010, Article ID 984927, 12 pages, 2010.

[33] M. Do Rosario De Pinho, M. M. Ferreira, U. Ledzewicz, and H. Schaettler, "A model for cancer chemotherapy with state-space constraints," Nonlinear Analysis, Theory, Methods and Applications, vol. 63, no. 5-7, pp. e2591-e2602, 2005.

[34] J. Llibre and M. Messias, “Global dynamics of the Rikitake system," Physica D. Nonlinear Phenomena, vol. 238, no. 3, pp. 241-252, 2009.

[35] Z. Wu and F. Ben Amara, "Parameterized regulator synthesis for bimodal linear systems based on bilinear matrix inequalities," Mathematical Problems in Engineering, vol. 2008, Article ID 341720, 22 pages, 2008.

[36] T.-C. Lin and M. Roopaei, "Based on interval type-2 adaptive fuzzy $\mathrm{H}^{\infty}$ tracking controller for SISO time-delay nonlinear systems," Communications in Nonlinear Science and Numerical Simulation, vol. 15, no. 12, pp. 4065-4075, 2010.

[37] L. Boutat-Baddas, J. P. Barbot, D. Boutat, and R. Tauleigne, "Sliding mode observers and observability singularity in chaotic synchronization," Mathematical Problems in Engineering, no. 1, pp. 11-31, 2004.

[38] Y.-J. Liu and N. Zhou, "Observer-based adaptive fuzzy-neural control for a class of uncertain nonlinear systems with unknown dead-zone input," ISA Transactions, vol. 49, no. 4, pp. 462-469, 2010.

[39] D. M. K. N. Kunitaki, B. S. L. P. De Lima, A. G. Evsukoff, and B. P. Jacob, "Probabilistic and fuzzy arithmetic approaches for the treatment of uncertainties in the installation of torpedo piles," Mathematical Problems in Engineering, vol. 2008, Article ID 512343, 26 pages, 2008.

[40] S. Tong and Y. Li, "Observer-based fuzzy adaptive control for strict-feedback nonlinear systems," Fuzzy Sets and Systems, vol. 160, no. 12, pp. 1749-1764, 2009.

[41] M. R. Hajj, M. Ghommem, and A. H. Nayfeh, "Control of limit cycle oscillations of a two-dimensional aeroelastic system," Mathematical Problems in Engineering, vol. 2010, Article ID 782457, 13 pages, 2010.

[42] F.-W. Xie, Y.-f. Hou, Z.-P. Xu, and R. Zhao, "Fuzzy-immune control strategy of a hydro-viscous soft start device of a belt conveyor," Mining Science and Technology, vol. 19, no. 4, pp. 544-548, 2009.

[43] H. Izadinia, F. Sadeghi, and M. M. Ebadzadeh, "A novel multi-epitopic immune network model hybridized with neural theory and fuzzy concept," Neural Networks, vol. 22, no. 5-6, pp. 633-641, 2009.

[44] W. Jiang, Q. Guo-Dong, and D. Bin, “Observer-based robust adaptive variable universe fuzzy control for chaotic system," Chaos, Solitons and Fractals, vol. 23, no. 3, pp. 1013-1032, 2005. 


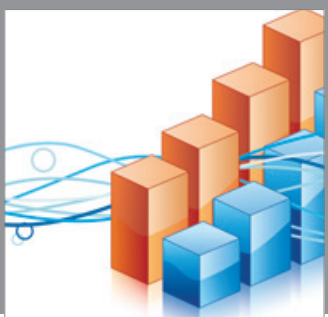

Advances in

Operations Research

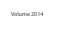

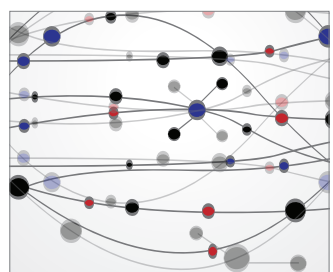

\section{The Scientific} World Journal
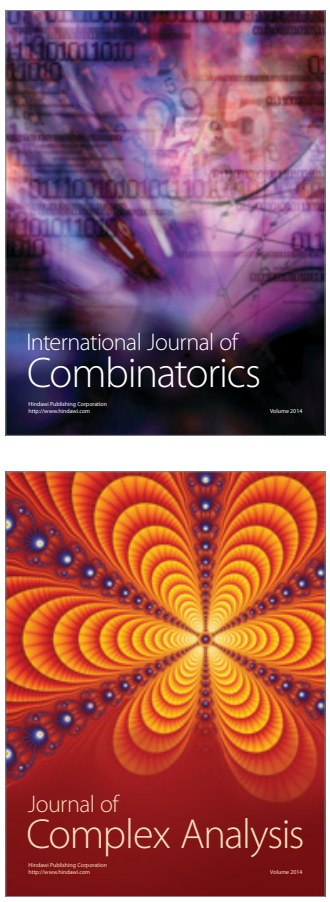

International Journal of

Mathematics and

Mathematical

Sciences
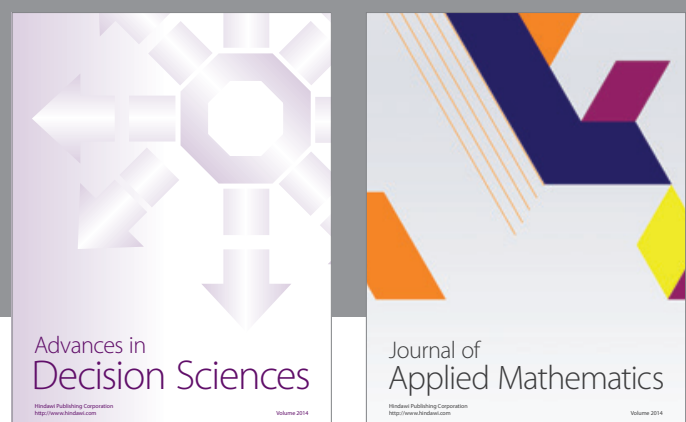

Journal of

Applied Mathematics
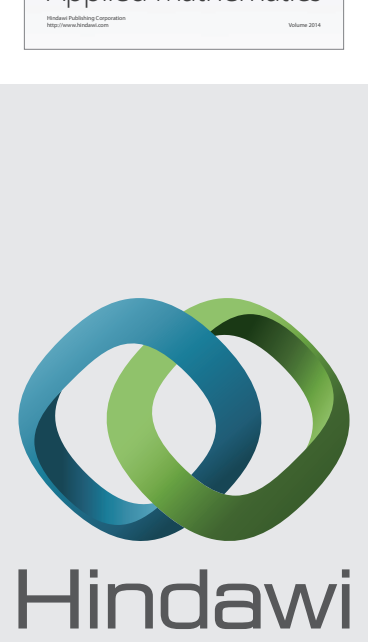

Submit your manuscripts at http://www.hindawi.com
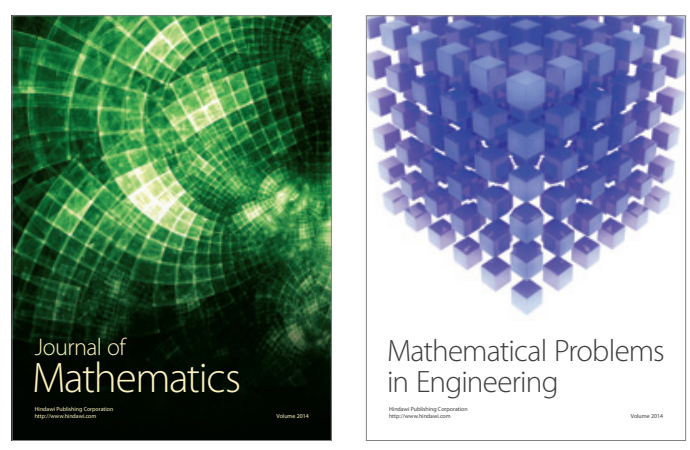

Mathematical Problems in Engineering
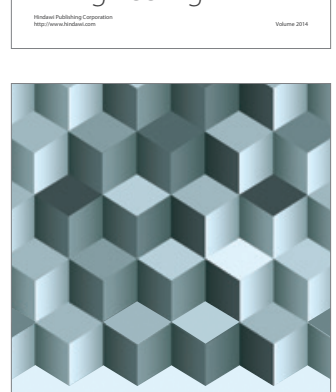

Journal of

Function Spaces
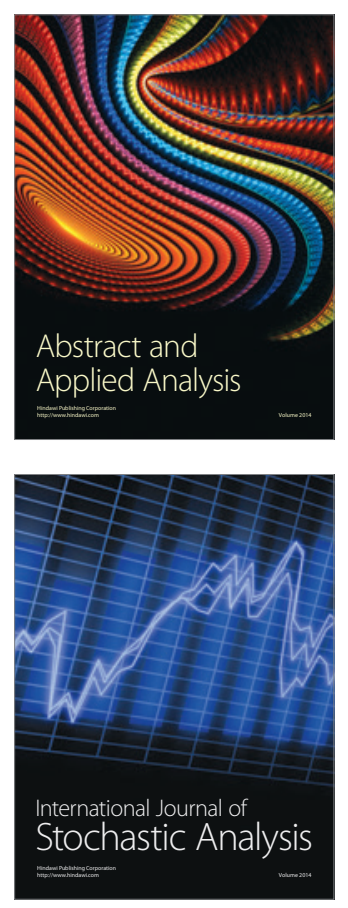

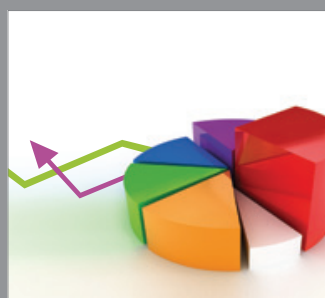

ournal of

Probability and Statistics

Promensencen
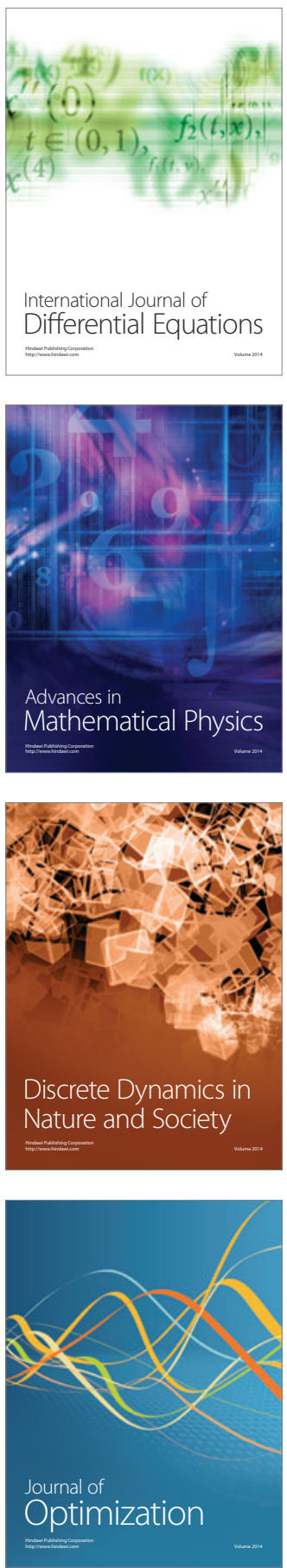